\title{
Acceptability and Impact of a Breathing Awareness Meditation Application on Stress, Strain, Sleep Quality Among Caregivers of Kidney Transplant Recipients
}

Jessica Chandler ${ }^{1 *}$, Samantha Pairet ${ }^{1}$, Kinsey N Kellam ${ }^{1}$, Allison Neely ${ }^{1}$, Luke R Sox ${ }^{1}$, Frank A Treiber ${ }^{1}$, and John W McGillicuddy ${ }^{2}$

${ }^{1}$ College of Nursing, Medical University of South Carolina, USA

${ }^{2}$ College of Medicine, Department of Surgery, Medical University of South Carolina, USA

*Corresponding author: Jessica Chandler, Medical University of South Carolina, 99

Jonathon Lucas Street, Charleston, SC, USA.

Received Date: September 05, 2020

Published Date: October 06, 2020

\section{Abstract}

Background: Caregivers are a crucial part of positive health outcomes for many chronically ill patients. Many caregivers experience stress and strain related to their caregiving role that may lead to neglecting their own health, both physical and mental, ultimately causing development of their own chronic illnesses.

Aim of the study: To evaluate acceptability and usability of a previously validated smartphone stress reduction app (Tension Tamer [TT]) among caregivers of kidney transplant recipients (KTR-Cs). An exploratory aim was to determine preliminary signals of efficacy of perceived stress, sleep quality, and blood pressure control.

Methods: A proof of concept randomized controlled trial with 20 KTR-Cs randomized into one of two groups: TT intervention arm or attention control (AC) arm. TT arm received a previously validated breathing awareness meditation (BAM) mobile application to use twice daily for three months while the AC arm received daily healthy education SMS messages. Study visits were conducted at baseline, 1-month, 2-month and 3-months visits.

Results: Mean age of the entire sample was $56.2 \pm 14.6$ years, $72.7 \%$ female and $59.1 \%$ African American (40.9\% white). Respondents averaged $19.3 \pm 11.7$ hours of caregiving/week, with $60 \%$ working full or part-time. There was a $100 \%$ participation rate and $90 \%$ retention rate. The majority of the sample (75\%) at baseline self-reported high perceived stressed, $90 \%$ indicated poor sleep and $60.0 \%$ had high caregiver strain. Average adherence across the trial to the TT protocol (two 10 min sessions/day) was $71.8 \%$. Compared to the AC group, the TT group experienced significantly greater improvements in perceived stress, caregiver strain, sleep quality and systolic blood pressure at $3-\mathrm{months}(-8.7 \mathrm{vs}+4.1 \mathrm{mmHg}$, $\mathrm{p}<.05)$.

Conclusion: Results from this study indicate that a user friendly, BAM program may be efficacious in improving perceived stress, sleep quality and blood pressure control among KTR-Cs if tested in a properly powered RCT.

Key words: Caregiver; Stress; Strain; Sleep; Breathing awareness meditation; Mobile health; Blood pressure

Abbreviations: KTR-C: Caregiver of kidney transplant recipient; MBSR: Mindfulness based stress reduction; mHealth: Mobile health; TT: Tension Tamer; BAM: Breathing Awareness Meditation; MBRT: Mindfulness based resilience training; AC: Attention Control; PSS: Perceived Stress Scale; CSI: Caregiver Strain Index; PSQI: Pittsburgh Sleep Quality Index; HTN: Hypertension; SBP: Systolic Blood Pressure

\section{Introduction}

Kidney transplantation offers patients with end stage renal disease the chance for a longer, higher quality of life [1]. To help achieve optimal results, transplant programs require wait-listed patients to identify a primary caregiver to assist throughout the 
demanding pre--and post-transplant process. Although the research on solid organ transplant (e.g., kidney, liver, and pancreas) caregiving is relatively sparse, similar to studies involving caregivers of other chronic disease patients (e.g., Alzheimer's, Parkinson's, cancer, etc.), findings consistently indicate that these type of caregivers also experience substantial caregiver strain and stress [2,3]. Intuitively, one may assume that post-transplant, kidney transplant recipient caregivers (KTR-Cs) often report a sense of relief due to their loved one's new lease on life (i.e., new organ). However, KTRCs remain heavily relied upon post-transplant. Several studies reported that contrary to expectations, perceived strain and quality of life of KTR-Cs did not improve from pre-transplant to 6-months post transplantation $[2,4]$. The continued strain on KTR-Cs is due to extensive and exhaustive tasks such as providing transportation to checkups, emotional support, monitoring health status, managing medication regimen, acute rejection episodes, graft failures, etc. Similar to caregivers of chronically ill and transplant patients (e.g., Alzheimer's, Parkinson's, heart, liver transplant), KTR-Cs often experience significant caregiving strain characterized by increased perceived stress, fatigue, sleep disturbance and/or anxiety, which, if prolonged, may lead to both chronic mental and physical health issues [2].

Several types of meditative programs including mantra, yogic, Mindfulness Based Stress Reduction (MBSR) and Benson relaxation technique implemented with caregivers of chronically ill patients (e.g., Alzheimer's, dementia, pre- and post- heart, bone marrow, liver and kidney transplant) have resulted in significant improvements in perceived stress, caregiver strain, quality of life, and sleep quality [5-10]. Collectively, these six studies utilized program delivery tactics ranging from in-person instruction to online modules and a combination of both. Duration of the interventions ranged four to 12 weeks and sample sizes ranged from nine to 150 participants. The majority of studies utilized versions of MBSR, as well as mantra and yogic meditation and the Benson relaxation technique (diaphragmatic breathing). Significantly greater improvements compared to standard care groups were noted in self-reported stress levels, caregiver strain and affective state (e.g., anxiety, depression) across the trials. However, those program reporting high degrees of success often included a substantial time investment from participants including travel for in person training sessions ( $\sim 2$-3 hours) and 8-12 weekly individual or group classes ( $\sim 1$ hour) and/or weekly online classes or practice. Majority of the interventions involved multi-method tactics and significant time requirements (e.g., wide array of topics presented across inperson or online modules ranging from 'putting yourself first', 'just breath', 'mindfulness and sleep', to 'eating mindfully' or, in-person sessions with each session including educational, experiential and mindful movement aspects plus a CD and written educational information for home practice sessions, etc.). The amount of time devoted to attending the various sessions, much less driving time if sessions were in person, as well as the potential overload of so many skills to learn and practice may have contributed to the 20 - $45 \%$ attrition rates in these trials that ran only 4 to 12 weeks. In summary, meditative programs are beneficial in addressing stress and strain experienced by caregivers but more user friendly delivery approaches are needed, along with tailoring of programs for specific needs and issues experienced by the particular caregiver group in order to reduce attrition rates and sustain participants' engagement over time.

Digital health enabled solutions are increasingly being shown to be successful in helping patients increase health promoting behaviors (e.g., breathing meditation, physical activity, diet, smoking cessation) and adherence to medical regimen [11-14]. A recent review of mobile apps designed for caregivers found that most apps target the needs of the patient being cared for while only $23 \%$ focused on supporting the caregivers' emotional needs (e.g., stress, anxiety, and depression) [3]. Although meditation programs were noted as being used, they were used with the patients and none were tailored specifically towards caregivers, and their unique stressors and experiences, even though there is evidence that tailored mobile health (mHealth) programs provide optimal results $[3,10]$.

Our work and that of others using theory-based, patient/ provider guided, iterative design has experienced high patient receptivity to digital technology enabled primary and secondary disease prevention self-management programs [11,15-17]. Pertinent to the current study, our team used a theory guided, user centered design approach to develop and test a smart phone stress reduction app (Tension Tamer [TT]) via NHLBI R01 funding [18]. The TT app delivers breathing awareness meditation (BAM), tracks adherence to sessions in real time via continuous heart rate acquisition using the video camera lens and reflective photoplethysmography $[18,19]$. TT users held one finger to the back of their smartphone camera to allow the app to acquire heart rate while engaging in BAM. Our most recent trials evaluating the TT app [20-22] corroborate and extend earlier traditional in person delivery of BAM sessions which, collectively have consistently observed significantly greater improvements in perceived stress, sleep quality and blood pressure levels among pre-hypertensive and hypertensive adolescents and adults compared to standard care groups [23-26].

The TT app was recently adapted for use among sarcoidosis patients with elevated stress and fatigue levels. Compared to a standard care control group, the TT group exhibited significantly greater reductions in perceived stress and fatigue over a threemonth acceptability trial [27]. Importantly, post-trial interviews indicated need for tailoring and inclusion of sarcoidosis-centered educational modules and testimonials [27]. Interviews also indicated several participants experienced difficulty in maintaining proper finger placement on the video camera lens during sessions due to finger clubbing, tremor and or excessive heat from the lens. 
Mass commercialization and consumer availability of wearable activity trackers with heart rate acquisition technology provided an opportunity to update the original TT app. This resulted in refinement of the TT app to incorporate the Fitbit Versa accelerometer for heart rate acquisition. This allows a more "hand off" approach for the user that may result in greater relaxation and focus upon BAM. In closing, the recently adapted TT app appears to be a potentially viable tactic for addressing KTR-C stress, strain and sleep quality.

As noted earlier, there have been limited empirical efforts to assess issues of stress, caregiver strain, and sleep quality among KTR-Cs. The only published study that we are aware of that included KTR-C's also included caregivers of heart, liver, pancreas and stem cell transplant recipients [8]. In addition, the study included the transplant recipients as part of the study population. While authors found promising results using mindfulness based resilience training (MBRT) on perceived stress, depression and anxiety, it is impossible to differentiate the effects the program had on the KTR-Cs, as the data were merged together across subgroups rather than stratified by types of participants (e.g., caregivers vs. patients, kidney vs heart vs liver transplant recipients, etc.) Therefore, drawing conclusions regarding effects of an in-person delivered MBRT program for any subgroup in the overall sample was not feasible.

To our knowledge, a smart phone delivered stress reduction app has not been developed with guidance from KTR-Cs. Capitalizing on our earlier work with the TT app, we are able to provide KTRCs experiencing elevated stress with an adaptation (inclusion of continuous heart rate acquisition via Fitbit Versa) of a smart phone BAM app shown to be user friendly, efficacious in reducing perceived stress, improving sleep quality and blood pressure levels among those who are prehypertensive or hypertensive [20-22]. The purpose of this study was to evaluate the acceptability and usability of a previously validated smartphone stress reduction app interfaced with a smart watch among KTR-Cs. An exploratory aim was to determine preliminary signals of clinical efficacy with regard to improvements in perceived stress, sleep quality, and blood pressure control.

\section{Materials and Methods}

\section{Study Population}

Eligible participants included adult caregivers of KTRs at any stage post-transplant receiving care at the Medical University of South Carolina (MUSC) Transplant Center. The Transplant Center is the sole transplant service provided in the state of South Carolina. There were no other exclusion criteria to participate. Caregivers were approached in the MUSC transplant clinic waiting room and asked if they would share their thoughts and experiences related to serving as a KTR-C via RedCap survey. Recruitment began June 2019 and ended August 2019. A study staff member and kidney transplant center nurse announced the opportunity to volunteer to take part in a quality improvement project, and the first 20 volunteers were enrolled. This quality improvement study was granted exemption by the MUSC Institutional Review Board.

\section{Study Design}

20 KTR-C's were enrolled into this 3-month, two arm proof of concept randomized controlled trial. Participants were randomly assigned into either the TT group or the attention control (AC) group.

Tension tamer intervention group: TT is a patient-centered, iteratively designed BAM application with content developed and refined guided by self-determination and social cognitive theories. A detailed description of all TT features is provided elsewhere $[19,20]$. TT was originally developed for pre-hypertensive adults and tested in a 6-month dose-response trial and a subsequent two-arm, randomized controlled trial that included 60 and 80 prehypertensive adults, respectively. found TT efficacious in reducing perceived stress, improving sleep quality and improving blood pressure control among pre-hypertensive adults [20-22].

The TT app was originally designed to use a smartphone's camera lens to acquire continuous heart rate via photoplethysmography. Continuous heart rate acquisition during sessions not only provides an objective measure of duration adherence to the BAM sessions but also enables verification of participants' transition into respiratory sinus arrhythmia resulting in a decreased heart rate [19]. However, feedback from the two arm TT trial, indicated that for some participants, holding their index finger to the camera's lens and light proved problematic, as some of the older patients experienced tremor and/or clubbing of the fingertip making heart rate detection difficult. Additionally, patients with older Android phones expressed discomfort from the phone's camera light heating up during heart rate detection. Therefore, with mass commercialization and widespread availability of wearable technology with the ability to detect continuous heart rate, we opted to integrate the Fitbit Versa watch with our TT app to provide a more "hands off" approach during TT sessions. This newly integrated version of the TT app was the version provided in this trial. Lastly, as with the earlier TT trials, after each TT session, the participant received an automatic, immediate feedback graph charting their average heart rate each minute across the 10-minute session.

During the baseline visit, the TT app was downloaded onto each participants' phone and a Fitbit Versa was loaned for the duration of the program. Study staff demonstrated how to use the watch and led the participants through a TT session. As the participants' practiced a TT session, study staff provided additional guidance and feedback as necessary (e.g., deep belly breathing rather than shallow breaths, keep eyes closed, lie down if they are able, etc.). Once they concluded the practice session with help from the study staff, each participant completed their first TT session on their own, demonstrating ability to participate without the staff's active 
help and guidance.

As noted earlier, TT was previously tested in a dose-response trial [20]. The optimal dosage across the 6 months to maximize adherence and primary outcome of reduced systolic blood pressure (SBP), was found to be 10-minute sessions, twice daily. For this trial, as was done in the recent sarcoidosis trial [27], we used the same regimen. Adherence scores were calculated daily dependent upon the previous day's TT activity. If a participants participated in 2, 10 -minute sessions, they received a 1.0 for that day. If a participant completed only 1, 10-minute session, they received a 0.5 score, and if they completed zero sessions, they received a 0.0 for that day. A daily positive feedback SMS message was automatically sent based upon the previous day's levels of adherence (complete, partial, none). For instance, if a participant received a 1.0 for the previous day, their feedback text message the next day might read "Great work on completing your two BAM sessions! Two BAMs a day helps keep the stress away" while a participant who engaged in zero sessions may receive a message reading "Looks like you had a busy day yesterday! Life can be hectic. It's important to find time to practice Tension Tamer twice each day to help reduce stress from such hectic times."

5.2.2.Attention Control (AC) Group: The AC group received daily SMS messages; however content was healthy lifestyle-behavior education messages associated with topics such as heart-healthy diet, smoking cessation, physical activity, sun exposure and sleep. An example message received by an AC participant might read "For better sleep, cut caffeine by the afternoon. Your caffeine jolt stays in your system longer than you might think" or "If possible, avoid sun exposure during the hours of $10 \mathrm{am}$ to $4 \mathrm{pm}$. It's is the best way to protect against harmful UV light." Other than these daily messages, the AC group were not asked to do anything else other than the monthly study visits to complete questionnaires and blood pressure measurements.

\section{Study assessments and time points}

A survey was adapted from previously validated questionnaires to assess demographics and caregiver information (hours/week involved in caregiving tasks, years spent as a caregiver, etc.) and administered to all KTR-C participants at their baseline study visit. Additionally, each participant completed the Perceived Stress Scale (PSS) [28], Caregiver Strain Index (CSI) [29], and the Pittsburg Sleep Quality Index (PSQI) [30] at four study visits across the trial: Baseline, 1-month, 2-month and 3-month visits. Resting blood pressures were measured at baseline and at the 3-month visit using a standardized protocol involving 3 measurements within a 10 -minute window (i.e., 1 st measurement at $0 \mathrm{~min}, 2 \mathrm{nd}$ at 5 min and 3rd at $7 \mathrm{~min}$ ) with the second and third measurements averaged to create the final value [20]. Lastly, we assessed the TT group's general perceptions of the TT program and usefulness in improving health outcomes at the 3-month visit.

\section{Data Analysis}

Clinical and demographic data were first examined for central tendencies (mean, median and range where applicable), then compared by group assignment using a pooled t-test for continuous variables and a X2/Fischer's Exact test for categorical variables. Pre/Post treatment within-group differences in continuous variables were assessed using paired sample t-tests. Statistical significance was set at $\mathrm{p}<.05$.

\section{Results}

We recruited 20 KTR-C's and randomly assigned them to receive the TT program $(n=10)$ or the AC program $(n=10)$. Two attention control participants were lost to follow up (i.e., we couldn't reach them to schedule their 1-month visit). All TT participants completed all study visits. Mean age of the entire sample was $56.2 \pm 14.6$ years with $72.7 \%$ female and 59.1\% African American (40.9\% white). Respondents averaged $19.3 \pm 11.7$ hours of caregiving/week, with $60 \%$ working full or part time jobs. Using established clinical cutoffs for the Perceived Stress Scale (i.e., $\geq 16$ on the PSS Scale [28]), $75.0 \%$ self-reported high perceived stressed, $90 \%$ indicated poor sleep using the Pittsburgh Sleep Quality Index (>5) [30] and 60.0\% had high degree of caregiver strain using the Caregiver Strain Index( $\geq 7$ ) [31]. The top two chronic physical diseases reported for KTR-Cs were hypertension (55.0\%) and diabetes (25.0\%). Table 1 presents demographics and caregiver characteristics separated by study arm.

Perceived stress and sleep quality significantly improved for the TT group between baseline and Month 3. Figure 1 illustrates the average group changes in perceived stress and Figure 2 illustrates average group changes in sleep quality by group across all time points. Table 2 summarizes caregiver strain results by group and time point. On several outcome parameters, significant differences were observed between groups at baseline and persisted across the trial. The TT group experienced higher caregiver strain at all time points compared to their attention control counterparts. While the TT arm began with higher strain, they experienced significant reductions in strain (-2.3 at month $1,-3.0$ at month 2 and -2.1 at month 3 , all ps<.05), although remaining higher than the attention control group at all time points. The attention control group's change in Caregiver Strain Index score from baseline to the 3-month time point was not significant $(\mathrm{p}=.17)$. This was not unexpected due to their significantly lower baseline value compared to the TT group ( $\mathrm{p}<.008)$. Lastly, the TT intervention group showed an overall reduction in SBP of $8.7 \mathrm{mmHg}$ from baseline to month 3 compared to a $4.1 \mathrm{mmHg}$ increase among the attention control arm $(\mathrm{p}<.001)$. These SBP findings align with our recent publications which found the TT app helpful in reducing SBP among those with hypertension (HTN) and pre-HTN [20,22].

Overall acceptability was good with a $100 \%$ participation rate and high retention rate of $90 \%$. The two individuals that 
dropped out of the study were in the SC group. Usability was good, as indexed by adherence rates to the twice per day dosage of 10-minute TT doses. The cumulative monthly adherence rates ranged from 73.4 - 85.8\% (M1: 85.8, M2: 81.0, M3: 73.4\%) giving an average adherence rate for the TTs across trial of $71.8 \%$. This met the desired adherence cut off level of $>75 \%$ [20]. On the first 2 months and was close for month 3 . The TT group exceeded the desired adherence threshold throughout the first two months and came close on the third. Furthermore, examination of individual participant adherence rates across the trial ranged from 46.8-

Table 1: Demographic Characteristics of Participants.

\begin{tabular}{|c|c|c|c|}
\hline & Tension Tamer $(n=10)$ & Attention Control $(n=10)$ & $t / X^{2} p$-value \\
\hline Age (years) & $58.4 \pm 3.7$ & $54.1 \pm 5.9$ & 0.46 \\
\hline \multicolumn{4}{|c|}{ Sex } \\
\hline Male & $50 \%$ & $30 \%$ & \\
\hline Female & $50 \%$ & $70 \%$ & \\
\hline \multicolumn{3}{|c|}{ Race } & 0.82 \\
\hline Non-Hispanic Black & $50 \%$ & $60 \%$ & \\
\hline Non-Hispanic White & $50 \%$ & $40 \%$ & \\
\hline \multicolumn{3}{|c|}{ Marital Status } & 0.37 \\
\hline Married/Living with Significant Other & $80 \%$ & $60 \%$ & \\
\hline Never married & $10 \%$ & $20 \%$ & \\
\hline Divorced/Separated & $10 \%$ & $10 \%$ & \\
\hline Widowed & $0 \%$ & $10 \%$ & \\
\hline \multicolumn{3}{|c|}{ Education } & 0.65 \\
\hline College Degree or above & $50 \%$ & $30 \%$ & \\
\hline Trade/Tech School & $10 \%$ & $30 \%$ & \\
\hline High School/GED & $40 \%$ & $40 \%$ & \\
\hline \multicolumn{3}{|c|}{ Work Status } & 0.56 \\
\hline Part/Full Time & $60 \%$ & $60 \%$ & \\
\hline Retired or Disabled & $40 \%$ & $40 \%$ & \\
\hline Average \# of people in household (n) & $3.2 \pm 0.45$ & $2.8 \pm 0.36$ & 0.38 \\
\hline Hours spent caregiving/week & $19.1 \pm 3.8$ & $14.6 \pm 7.3$ & 0.62 \\
\hline \multicolumn{3}{|c|}{ Years of Caregiving } & 0.88 \\
\hline$<1 \mathrm{y}$ & $10 \%$ & $30 \%$ & \\
\hline $1-4 y$ & $50 \%$ & $30 \%$ & \\
\hline$\geq 5$ years & $40 \%$ & $40 \%$ & \\
\hline
\end{tabular}

Table 2: Caregiver Strain Index Scores by Group Across the 3-month Trial.

\begin{tabular}{|c|c|c|c|c|}
\hline Time point & Tension Tamer (mean score) & High Strain (n) & Attention Control (mean score) & High Strain (n) \\
\hline Baseline & 8.2 & 9 & 3.9 & 3 \\
\hline Month 1 & $5.9^{*}$ & 5 & 4.4 & 3 \\
\hline Month 2 & $5.2^{*}$ & 3 & 4.4 & 2 \\
\hline Month 3 & $6.1^{*}$ & 5 & 3.8 & 2 \\
\hline
\end{tabular}

*Indicates within group statistical significance compared to baseline values $p<.05$

Table 3: Tension Tamer Acceptability Responses from TT group $(n=10)$. 
2. My physical health is better than it was before I used the Tension Tamer system.

3. I am more involved in helping myself stay healthy using the Tension Tamer system.

4. The Tension Tamer system helps me to better manage my emotional health, especially related to stress.

5. The Tension Tamer system helps me to better manage my physical health related to stress exposure.

6. I follow my doctor's advice better since working with the Tension Tamer system.

7. The Tension Tamer smartphone and app are easy to use.

8. I can always trust the Tension Tamer app to work properly.

9. It was easy to learn how to use the Tension Tamer app.

10. My emotional health and well-being are better than before since using the Tension Tamer system.

11. The privacy of my heart rate data are protected over the internet.

12. I can effectively explain any problems I may be experiencing over the phone with my Tension Tamer navigator.

13. My Tension Tamer navigator answers my questions.

4.1

4.2

4.3

2.6

4.5

3.7

4.7

3.6

3.6

3.1

4.4

Scores are based on a Likert Scale from 1-5, Completely Disagree (1) - Neutral (3) - Completely Agree (5)

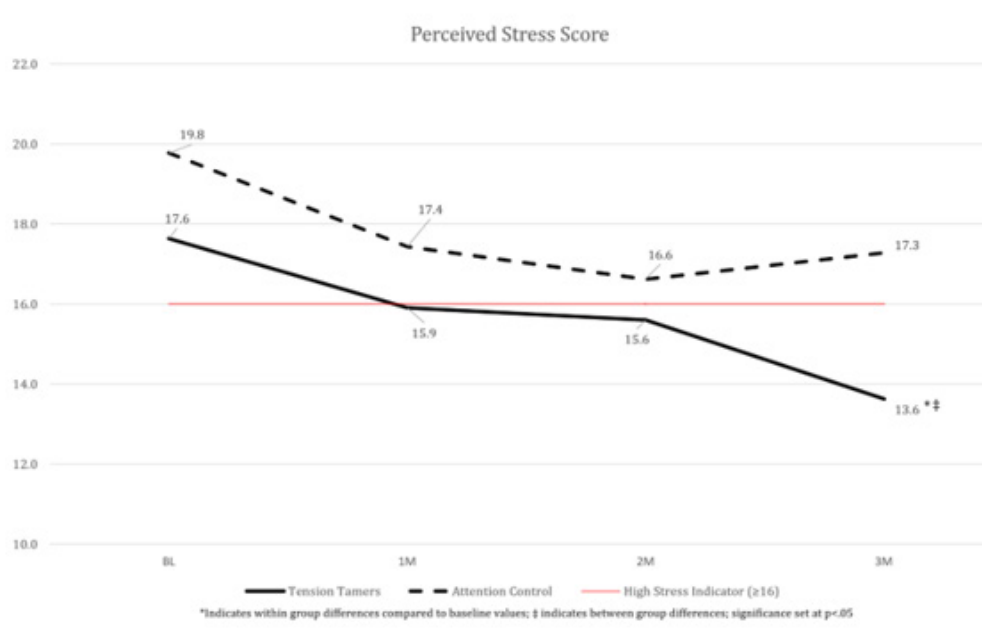

Figure 1: Group averages of perceived stress across study time points.

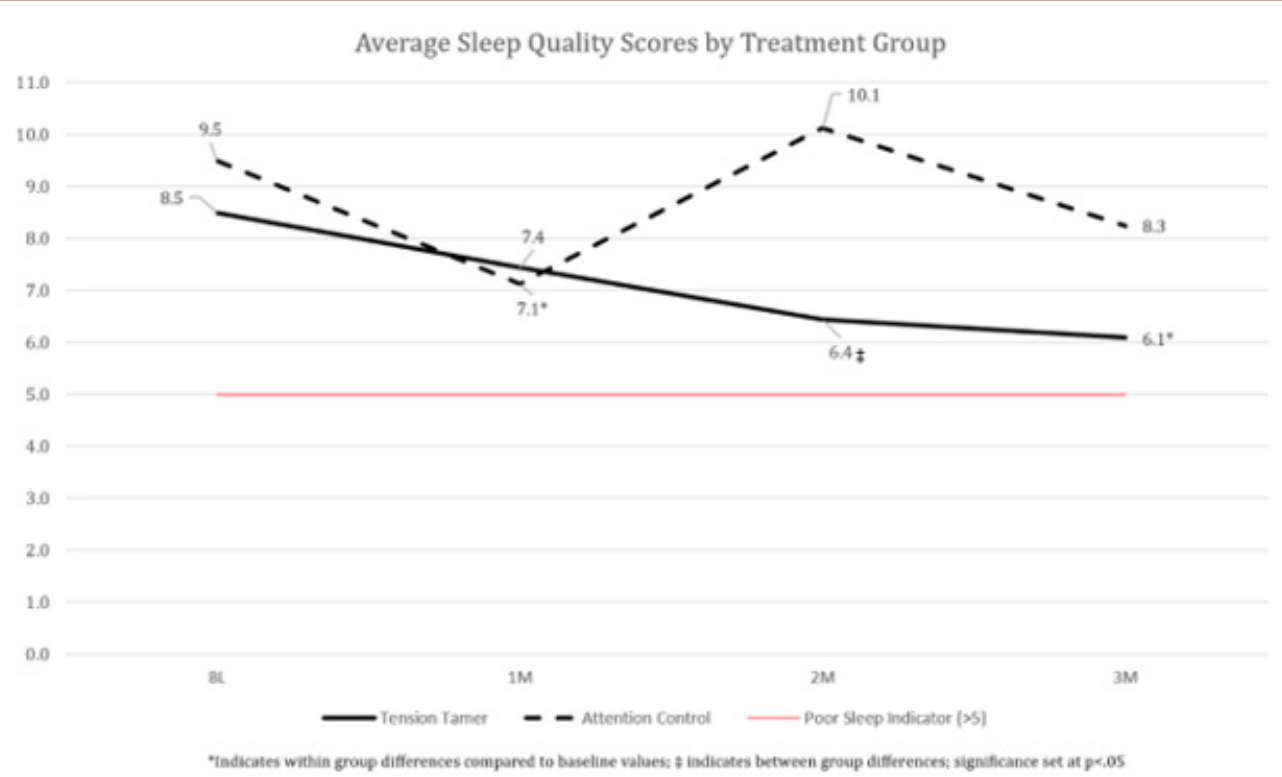

Figure 2: Group averages of sleep quality across study time points. 


\section{Discussion}

The current state of literature reporting on demographics, physical and mental health status, and overall quality of life among KTR-Cs is scarce. Using validated questionnaires with established clinical cutoff scores, $75.0 \%$ were experiencing elevated general stress levels, 90\% indicated poor sleep quality characterized by long latency, frequent awakening, etc.) and $60.0 \%$ had high degree of caregiver strain. However, it is neither unexpected nor surprising that KTR-Cs experience similar life stressors and strain compared to caregivers of other patient populations [2,3,6,3233]. Interestingly, $55 \%$ of KTR-Cs were diagnosed with hypertension and that those who engaged in the TT program showed clinically meaningful reductions in their SBP (i.e., $-13.5 \mathrm{mmHg}$ from baseline to month 3 for those diagnosed with HTN). Whether their SBP reductions were due to reductions in sympathetic drive due to TT's BAM program and/or an increase in their medication adherence levels is unclear. Evidence collected in this study further verifies the need for the transplant patients' healthcare management program to include assessment of the health and well-being of the KTR caregiver and if needed, providing access to appropriate services.

This 3-month, proof of concept RCT was found to have high acceptability with $100 \%$ participation rate and a $90 \%$ retention rate. This retention rate was much higher than that observed in the review of previous caregiver programs which lasted 1 -3 months. Possible reasons include that the BAM program was easy to learn, could be done at any time that fit best into their daily schedules and they received immediate physiological feedback of their heart rate changes during each session as well as brief SMS social reinforcement messages based upon levels of adherence. Usability of the TT program was also high as the group average for adherence to the TT regimen across the 3-month trial was $71.8 \%$. Broken down by month, the TT group adherence rates ranged from 73.4 $85.8 \%$. The desired adherence cut off level of $>75 \%$ [20] was met on the first 2 months and was close for month 3. In comparison to other mindfulness-based programs for caregivers, adherence to TT was on similar to, if not higher, than many studies, including our earlier work [6.22,32-34].

This study provides further evidence of BAM's effect on perceived stress and sleep quality. The TT group experienced significant improvements on the Perceived Stress Scale (PSS; -4.0 points) and sleep quality ( -2.4 points). Comparable PSS findings were reported in several caregiver studies (average of -5.6 points) $[32,33,35]$. To illustrate the clinical meaningfulness of the findings, 4 of the TT participants who were above clinical cutoff metrics on the PSS at baseline showed improvements dropping them into normal range for their age and race at the 3-month visit. Similarly, sleep quality categorizations shifted with 3 TT participants shifting from the poor sleep category to normal sleep.

Although, the TT group reported significantly greater reductions in caregiver strain, their scores remained consistently higher than the SC group at all time points from baseline through the 3-months. These findings may be partially explained by the immense difference ( $\sim 5$ points) between the two groups' strain scores at baseline (i.e., the TT group had more room for improvement,). Tkatch et al., reported significant improvements in caregiver strain as a result of an 8-week, 1-hour program mindfulness-based program [6]. Their explanation for these improvements included using a multifaceted approach with several objectives including mindfulness, education, and self-care. The self-care module included teaching selfcompassion, which the authors contributed to the significant reductions in caregiver burden. Before the TT app is evaluated in a properly powered efficacy RCT, we will assess need for the addition of various educational components to be viewed within the app (e.g., brief educational video clip links, testimonials, etc.) A user-center approach, including discussions with caregivers and transplant caregiving organizations, will help identify potentially useful topics that may enhance the impact of the KTR-C TT program upon caregiver strain.

Lastly, the TT group experienced clinically and statistically significant reductions $(-8.7 \mathrm{mmHg})$ in SBP after the three-month trial. For those diagnosed with hypertension, and even greater reduction was noted $(-13.7 \mathrm{mmHg})$ compared to the attention control group that showed a slight increase in SBP $(+4.1 \mathrm{mmHg})$. The current results corroborate and extend previous results in Stage 1 hypertensives who experienced a reduction of nearly $8 \mathrm{mmHg}$ after using TT for 3-months. These results are consistent to others using breathing techniques to improve BP control. Our results are comparable to other trials using breathing relaxation techniques, including BAM, which were often offered as part of a larger program and reported reductions ranging from 4.3 to $7.2 \mathrm{mmHg}$ [36-38]. Our participants experienced greater reductions, however they may have been more adherent to TT than participants of other trials, we had a large percentage of hypertensives in our TT cohort creating greater room for improvement, and our cohort started the trial with high degrees of perceived stress, strain and poor sleep quality, indicating great need for a stress reduction program and perhaps more motivation for participation.

\section{Conclusion}

The results of this acceptability trial indicate that KTR-Cs that experience stress, sleep disturbances, caregiver strain may experience improvements through an easy to use, smart phone delivered BAM program. Using a properly powered RCT, further testing is warranted to obtain estimates of effects size and strengthened signal of efficacy.

\section{Acknowledgements}

We thank the MUSC Transplant Center for giving us approval and support to conduct this quality improvement program. We also thank the Caregivers, first and foremost for being dedicated caregivers to their loved ones, and secondly for volunteering to take part in this project. 


\section{Conflict of Interest}

The authors have no conflicts of interests to declare.

\section{References}

1. (2019) Organ Procurement and Transplantation Network. National Data: Transplant by Donor Type. U.S. Department of Health \& Human Services April 9, 2019.

2. Rodrigue JR, Dimitri N, Reed A, Antonellis T, Pavlakis M, et al. (2010) Spouse caregivers of kidney transplant patients: quality of life and psychosocial outcomes. Prog Transplant 20(4): 335-342.

3. Grossman MR, Zak DK, Zelinski EM (2018) Mobile Apps for Caregivers of Older Adults: Quantitative Content Analysis. JMIR M health U health 6(7): e162.

4. Wicks MN, Milstead EJ, Hathaway DK, Cetingok M (1998) Family caregivers' burden, quality of life, and health following patients' renal transplantation. J Transpl Coord 8(3): 170-176.

5. Gross CR, Kreitzer MJ, Thomas W, Reilly-Spong M, Cramer-Bornemann M, et al. (2010) Mindfulness-based stress reduction for solid organ transplant recipients: a randomized controlled trial. Altern Ther Health Med 16(5): 30-38.

6. Tkatch R, Bazarko D, Musich S, Wu L, MacLeod S, et al. (2017) A Pilot Online Mindfulness Intervention to Decrease Caregiver Burden and Improve Psychological Well-Being. J Evid Based Complementary Altern Med 22(4): 736-743.

7. Dharmawardene M, Givens J, Wachholtz A, Makowski S, Tjia J (2016) A systematic review and meta-analysis of meditative interventions for informal caregivers and health professionals. BMJ Support Palliat Care 6(2): 160-169.

8. Stonnington CM, Darby B, Santucci A, Mulligan P, Pathuis P, et al. (2016) A resilience intervention involving mindfulness training for transplant patients and their caregivers. Clin Transplant 30(11): 1466-1172.

9. Lavretsky H, Epel E, Siddarth P, Nazarian N, Cyr NS, et al. (2013) A pilot study of yogic meditation for family dementia caregivers with depressive symptoms: effects on mental health, cognition, and telomerase activity. Int J Geriatr Psychiatry 28(1): 57-65

10. Ploeg J, Markle-Reid M, Valaitis R, McAiney C, Duggleby W, et al. (2017) Web-based interventions to improve mental health, general caregiving outcomes, and general health for informal caregivers of adults with chronic conditions living in the community: rapid evidence review. J Med Internet Res 19(7): e263.

11. McGillicuddy JW, Taber DJ, Mueller M, Patel S, Baliga PK, et al. (2015) Sustainability of improvements in medication adherence through a mobile health intervention. Prog Transplant 25(3): 217-223.

12. Gandhi S, Chen S, Hong L, Sun K, Gong E, et al. (2017) Effect of Mobile Health Interventions on the Secondary Prevention of Cardiovascular Disease: Systematic Review and Meta-analysis. Can J Cardiol 33(2): 219231.

13. Chandler J, Sox L, Kellam K, Feder L, Nemeth L, et al. (2019) Impact of a Culturally Tailored mHealth Medication Regimen Self-Management Program upon Blood Pressure among Hypertensive Hispanic Adults. Int J Environ Res Public Health 16(7): 1226

14. Debon R, Coleone JD, Bellei EA, De Marchi ACB (2019) Mobile health applications for chronic diseases: A systematic review of features for lifestyle improvement. Diabetes Metab Syndr 13(4): 2507-2512.

15. McGillicuddy JW, Weiland AK, Frenzel RM, Mueller M, Brunner-Jackson BM, et al. (2013) Patient attitudes toward mobile phone-based health monitoring: questionnaire study among kidney transplant recipients. J Med Internet Res 15(1): e6.

16. McGillicuddy JW, Gregoski MJ, Weiland AK, Rock RA, Brunner-Jackson BM, et al. (2013) Mobile Health Medication Adherence and Blood
Pressure Control in Renal Transplant Recipients: A Proof-of-Concept Randomized Controlled Trial. JMIR Res Protoc 2(2): e32.

17. Davidson TM, McGillicuddy J, Mueller M, Brunner-Jackson B, Favella A, et al. (2015) Evaluation of an mHealth Medication Regimen SelfManagement Program for African American and Hispanic Uncontrolled Hypertensives. J Pers Med 5(4): 389-405.

18. Gregoski MJ, Mueller M, Vertegel A, Shaporev A, Jackson BB, et al. (2012) Development and validation of a smartphone heart rate acquisition application for health promotion and wellness telehealth applications. Int J Telemed App 2012: 696324.

19. Gregoski MJ, Vertegel A, Shaporev A, Treiber FA (2013) Tension Tamer: delivering meditation with objective heart rate acquisition for adherence monitoring using a smart phone platform. J Altern Complement Med 19(1): 17-19.

20. Adams ZW, Sieverdes JC, Brunner-Jackson B, Mueller M, Chandler J, et al. (2018) Meditation smartphone application effects on prehypertensive adults' blood pressure: Dose-response feasibility trial. Health Psychol 37(9): 850-860.

21. Sieverdes JC, Treiber FA, Kline CE, Mueller M, Brunner-Jackson B, Sox LR, et al. Ethnicity Differences in Sleep Changes Among Pre-hypertensive Adults Using a Smartphone Meditation Application: A Dose-Response Trial. JMIR Protocols. in press.

22. Chandler J, Sox L, Diaz V, Kellam K, Neely A, et al. (2020) Impact of 12-Month Smartphone Breathing Meditation Program upon Systolic Blood Pressure among Non-Medicated Stage 1 Hypertensive Adults. Int J Environ Res Public Health 17(6): 1955.

23. Barnes VA, Orme-Johnson DW (2012) Prevention and Treatment of Cardiovascular Disease in Adolescents and Adults through the Transcendental Meditation(®) Program: A Research Review Update. CurrHypertens Rev 8(3): 227-242.

24. Barnes VA, Gregoski MJ, Tingen MS, Treiber FA (2010) Influences of Family Environment and Meditation Efficacy on Hemodynamic Function among African American Adolescents. J Complement Integr Med 7(1): 1326 .

25. Barnes VA, Pendergrast RA, Harshfield GA, Treiber FA (2008) Impact of breathing awareness meditation on ambulatory blood pressure and sodium handling in prehypertensive African American adolescents. Ethn Dis 18(1): 1-5.

26. Wright LB, Gregoski MJ, Tingen MS, Barnes VA, Treiber FA (2011) Impact of Stress Reduction Interventions on Hostility and Ambulatory Systolic Blood Pressure in African American Adolescents. J Black Psychol 37(2): 210-233.

27. James WE, Chandler JL, Kellam K, Neely A, Mount R, et al. (2019) Sarcoidosis Patients' Acceptability of Smartphone Application for Managing Stress and Fatigue. Annals of Behavioral Medicine. (in press).

28. Cohen S, Kamarck T, Mermelstein R (1983) A global measure of perceived stress. J Health Soc Behav 24(4): 385-396.

29. Robinson BC (1983) Validation of a Caregiver Strain Index1. J Gerontol 38(3): 344-348

30. Buysse DJ, Reynolds CF, Monk TH, Berman SR, Kupfer DJ (1989) The Pittsburgh Sleep Quality Index: a new instrument for psychiatric practice and research. Psychiatry res 28(2): 193-213.

31. Robinson BC (1983) Validation of a Caregiver Strain Index. J Gerontol 38(3): 344-348.

32. Haines J, Spadaro KC, Choi J, Hoffman LA, Blazeck AM (2014) Reducing stress and anxiety in caregivers of lung transplant patients: benefits of mindfulness meditation. Int J Organ Transplant Med 5(2): 50-56.

33. Whitebird RR, Kreitzer M, Crain AL, Lewis BA, Hanson LR, et al. (2013) Mindfulness-based stress reduction for family caregivers: a randomized controlled trial. Gerontologist 53(4): 676-686. 
34. Kubo A, Kurtovich E, McGinnis M, Aghaee S, Altschuler A, Quesenberry C, et al. (2019) A Randomized Controlled Trial of mHealth Mindfulness Intervention for Cancer Patients and Informal Cancer Caregivers: A Feasibility Study Within an Integrated Health Care Delivery System. Integr Cancer Ther 18: 1534735419850634.

35. Shapiro SL, Astin JA, Bishop SR, Cordova M (2005) Mindfulness-based stress reduction for health care professionals: results from a randomized trial. International journal of stress management 12(2): 164.

36. Wang S, Li S, Xu X, Lin G, Shao L, et al. (2010) Effect of slow abdominal breathing combined with biofeedback on blood pressure and heart rate variability in prehypertension. J Altern Complement Med 16(10): 10391045

37. Dickinson H, Campbell F, Beyer F, Nicolson D, Cook J, et al. (2008) Relaxation therapies for the management of primary hypertension in adults: a Cochrane review. Cochrane Database Syst Rev 22(12): 809-820.

38. Mourya M, Mahajan AS, Singh NP, Jain AK (2009) Effect of slow- and fastbreathing exercises on autonomic functions in patients with essential hypertension. J Altern Complement Med 15(7): 711-717. 